\title{
A Wide Axial Ratio Bandwidth Circularly Polarized Antenna for Spectrum Occupancy Measurement Campaign
}

\author{
Siddharudha Shivputra Shirgan ${ }^{1}$ and Uttam Laxman Bombale ${ }^{2}$ \\ Electronics and Telecommunication Dept., NBNSCOE, Solapur University, India ${ }^{1}$ \\ Electronics and Telecommunication Dept., DOT, Shivaji University, India ${ }^{2}$ \\ ${ }^{1}$ ssshirgan@gmail.com, ${ }^{2} u l b \_t e c h @$ unishivaji.ac.in
}

\begin{abstract}
The spectrum measurement campaigns conducted worldwide have confirmed by showing that the considerable amount of radio spectrum is underutilized. In the literature, it is observed that most of the spectrum measurement campaign utilizes commercially available antennas. In this communication, a novel circularly polarized wide axial ratio bandwidth circularly polarized microstrip antenna is designed for Spectrum Occupancy Measurement. The antenna exhibits, wide $-10 \mathrm{~dB}$ impedance bandwidth from $700 \mathrm{MHz}$ to $2.9 \mathrm{GHz}$ and wide $-3 \mathrm{~dB}$ axial ratio bandwidth from $1.5 \mathrm{GHz}$ to $2.7 \mathrm{GHz}$. The prototype of the antenna is fabricated, and the simulated results show very good agreement with measured results. Thereafter, the spectrum sensing ability of the antenna is verified through the spectrum sensing measurement setup show excellent spectrum sensing ability, -24 to -31.5 dBm in GSM $900 \mathrm{MHz}, \mathrm{LTE} 2.1 \mathrm{GHz}$, and ISM $2.4 \mathrm{GHz}$ bands in the outdoor surrounding. The proposed antenna is compact and easily portable and easy to integrate with the spectrum sensing measurement setup.
\end{abstract}

Keywords: Cognitive Radio, Circularly Polarized Antenna, Spectrum Measurement campaign, Spectrum occupancy, Ultra-wideband antenna

\section{Introduction}

Due to the technological development of various wireless communication systems and demand for wideband spectrum, the radio spectrum has become real estate for global communication. However, due to the licensing issue, the spectrum is not occupied for a continuous period thus, remains underutilized. Cognitive radio is an enabling technology where secondary users are allowed to use the unused licensed spectrum temporally in the absence of a primary user. In Cognitive Radio systems, spectrum sensing is performed using an ultra-wideband antenna, and then communication is established through narrowband/wideband antennas. Although, it is essential to understand the spectrum occupancy before exploitation of the licensed band. The idea of Cognitive Radio is extensively exploited in [1-2]. However, worldwide many researchers have exploited the inefficient utilization of the available frequency spectrum. The spectrum sensing measurement is done by M. Hoyhtyo et al., at Turku, Finland uses an RFeye receiver antenna, RF front end and data logger [3]. The spectrum occupancy measurement done in this above work is limited to $100 \mathrm{MHz}$ to the $800 \mathrm{MHz}$ band. In [4], M. Wellens et al., have used three different Discone antennas to cover $20 \mathrm{MHz}$ to $6 \mathrm{GHz}$ spectrum occupancy measurement which increase the overall complexity and cost of the measurement setup. Similarly, in [5] and [6], Discone antennas are utilized to identify the spectrum occupancy in Nigeria and Pune respectively. Even, almost all the spectrum occupancy measurement setup uses commercially available antennas for spectrum

Received (February 1, 2017), Review Result (August 15, 2017), Accepted (August 24, 2017) 
occupancy measurement for cognitive radio which are larger in size leading to a complex setup.

In this work, we propose a wide axial ratio bandwidth circularly polarized monopole microstrip antenna for the spectrum occupancy measurement, which will significantly reduce the overall cost of the measurement setup, installation complexity and easily portable. The circular polarization provides precise and accurate spectrum occupancy in the intended territory, and wide bandwidth enables reduces the requirement of multiple antennas. The performance of the antenna is verified by comparing the simulation and measurements results, and the spectrum occupancy measurement is demonstrated in indoor as well as outdoor surrounding at Solapur, India. In indoor surrounding, the proposed antenna senses $-35 \mathrm{dBm}$ power in LTE $2.1 \mathrm{GHz}$ and ISM $2.4 \mathrm{GHz}$ bands and $36 \mathrm{dBm}$ power in GSM $900 \mathrm{MHz}$ band. Following the introduction, the antenna design and analysis is presented in Section 2. In Section 3, simulated and measured results of the proposed antenna are presented. The Section 4 demonstrates and discusses the spectrum occupancy measurement setup and obtained results. Finally, the work is concluded in Section 5 .

\section{Antenna Design and Structure}

In wireless communication systems, the signal comes from different directions with a variety of polarization. Polarization is defined as the orientation of electromagnetic (EM) Field vectors at a given point and how it varies with time. According to the shape of the traces, three types of polarization exists for harmonic waves: linear, circular and elliptical, as shown in Figure 1.

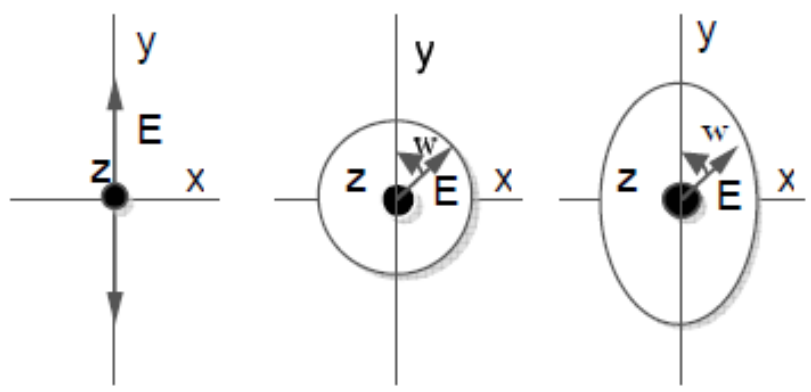

\section{Figure 1. Types of Polarizations: (a) Linear Polarization, (b) Circular Polarization and (c) Elliptical Polarization}

Naturally, the polarization of conventional antennas is not same as of the incident signals and introduces higher polarization mismatch resulting in a large polarization loss between transmitting and the receiving antenna. So for better mobility, weather penetration, flexibility in orientation angle and reduction in reflection, a polarization independent wireless communication system is required. The circularly polarized antennas are independent of the transmitter and receiver orientation and most commonly used in wireless communication systems [7]. To achieve the circularly polarized signals the signals must have two equal magnitude orthogonal components. That is by assuming the wave propagation in Z-direction the condition for circular polarization is given as [89]:

$$
\begin{aligned}
& |E x|=|E y| \\
& \emptyset_{\mathrm{x}}-\emptyset_{\mathrm{y}}=\left(\frac{1}{2}+n\right) \pi \text { for RHCP } \\
& \emptyset_{\mathrm{x}}-\emptyset_{\mathrm{y}}=-\left(\frac{1}{2}+n\right) \pi \text { for LHCP }
\end{aligned}
$$


Where, $\mathrm{n}=0,1,2,3,4 \ldots$

The various methods have been implemented to achieve the above conditions as dual orthogonal feed [10-11], by introducing modified patch radiator structures at the proper location on the feed point in a single feed antenna [12-13]. Single feed structures are more compact than a dual feed structure. The dual feed network requires a larger ground plane area for the feeding network but gives wideband response [14-15]. In this section, a wideband circularly polarized patch antenna is designed covering $700 \mathrm{MHz}$ to $2.7 \mathrm{GHz}$. Inspired from [16], a partial ground plane antenna is chosen as a basic structure to obtain the ultra-wideband response.
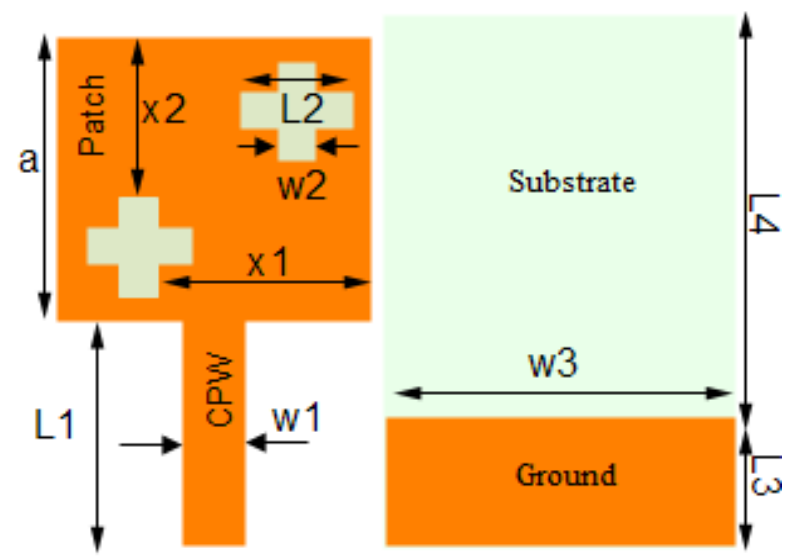

Figure 2. Configuration of the Slotted Antenna $(a=31 \mathrm{~mm}, \mathrm{~L} 1=17 \mathrm{~mm}$, w1= $2.38 \mathrm{~mm}, x 2=16 \mathrm{~mm}, x 1=21.75 \mathrm{~mm}, \mathrm{~L} 2=13 \mathrm{~mm}, w 2=3 \mathrm{~mm}, w 3=39 \mathrm{~mm}$, $L 3=11.8 \mathrm{~mm}$ and $L 4=40.2 \mathrm{~mm}$ )

Table 1. Structure of all Three Configurations (mm)

\begin{tabular}{lcccccc}
\hline Configuration & $\mathbf{W}$ & $\mathbf{L}$ & $\mathbf{W}_{\mathbf{g}}$ & $\mathbf{L g}_{\mathbf{g}}$ & $\mathbf{W}_{\mathbf{s}}$ & $\mathbf{L}_{\mathbf{s}}$ \\
\hline Conventional & 32 & 32 & 40 & 53 & NA & NA \\
\hline Partial Ground & 32 & 32 & 40 & 12 & NA & NA \\
\hline Slotted & 32 & 32 & 40 & 12 & 3 & 13 \\
\hline
\end{tabular}

The structure and dimensions of the proposed antenna are shown in Figure 2. The antenna is built on an FR4 Epoxy dielectric substrate of the dielectric constant of 4.4, a dielectric loss tangent of 0.02 and thickness of $1.6 \mathrm{~mm}$. The FR4 substrate is chosen due to the low-cost and ease of availability. However, better radiation performance can be obtained using the low dielectric material [17], as RT Duroid 5880 of dielectric constant 2.2. A Co-planer microstrip line feeding is provided through $50 \Omega$ SMA connector to excite the antenna. During the design and analysis, three different configurations: Conventional, Miniature Wideband and Cross-shaped slotted antennas are simulated. The structure of all three configurations is described in Table 1. 


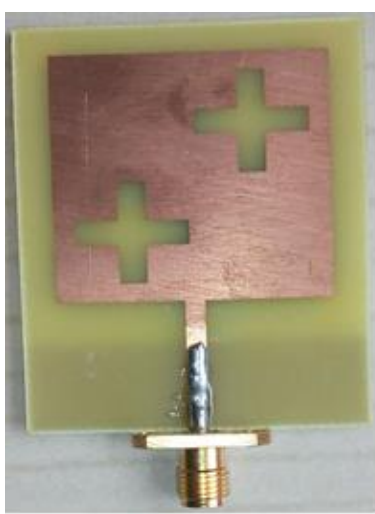

Top

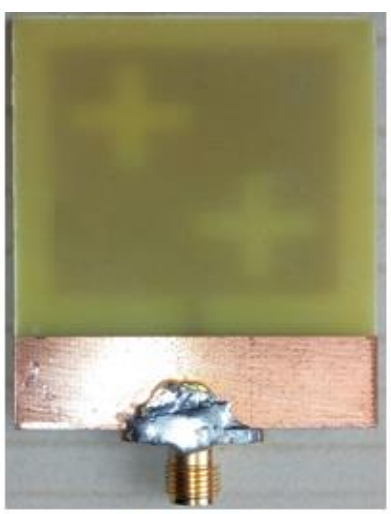

Bottom

Figure 3. Fabricated Prototype of Designed Antenna

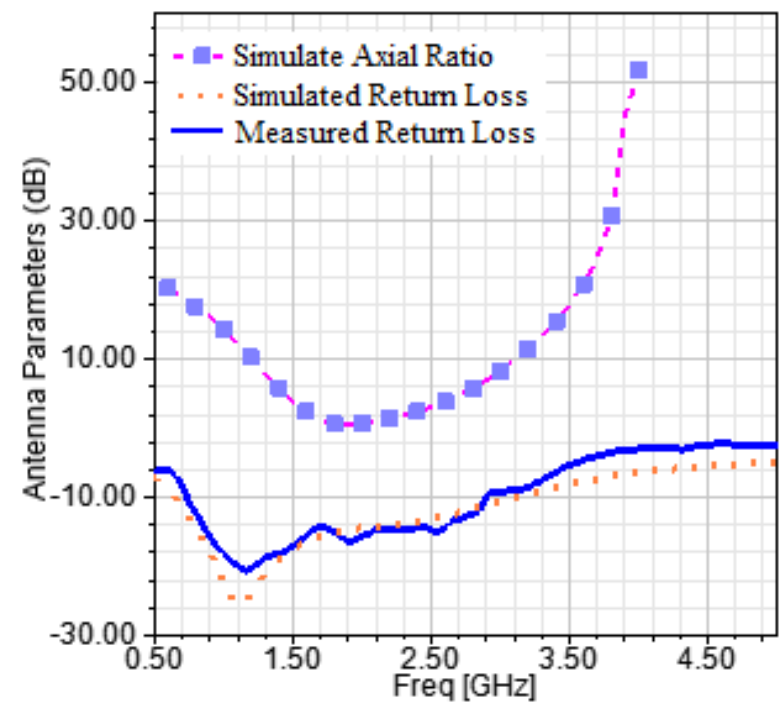

Figure 4. Return Loss and the Axial Ratio of Designed Antenna $\left(\varnothing=92^{\circ}\right.$, $\theta=0^{\circ}$ )

During experimentation, it is observed that an optimized partial ground plane results in an ultra-wideband response from $700 \mathrm{MHz}$ to $2.9 \mathrm{GHz}$. Thereafter, loading the crossshaped slots improves the axial ratio of the antenna and results in circular polarization with wide axial ratio bandwidth. The inclusion of symmetrical cross-shaped structures introduces orthogonal electric field vectors of equal magnitude, and thus circular polarization is obtained.

\section{Results and Discussion}

To achieve the miniature wideband antenna two different techniques have been implemented: use of monopole structure and loading of addition parasitic as a partial ground. A monopole antenna has a smaller size than dipole antennas. So the ground plane of the conventional antenna is removed which results in miniaturization. To enhance the 


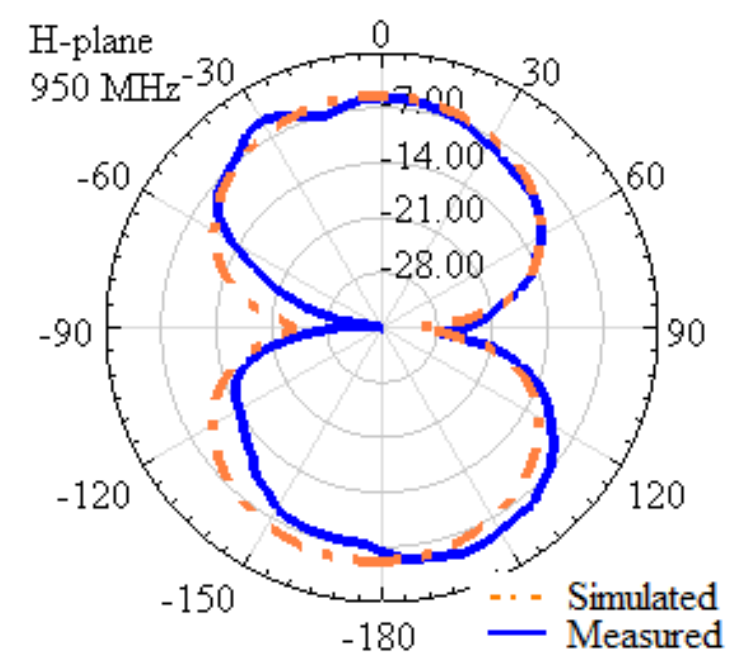

(a)

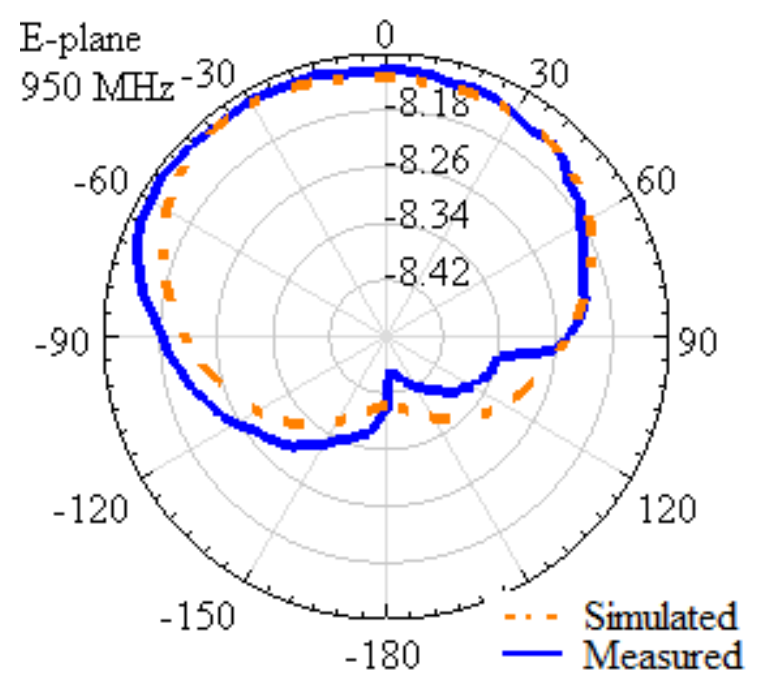

(b)

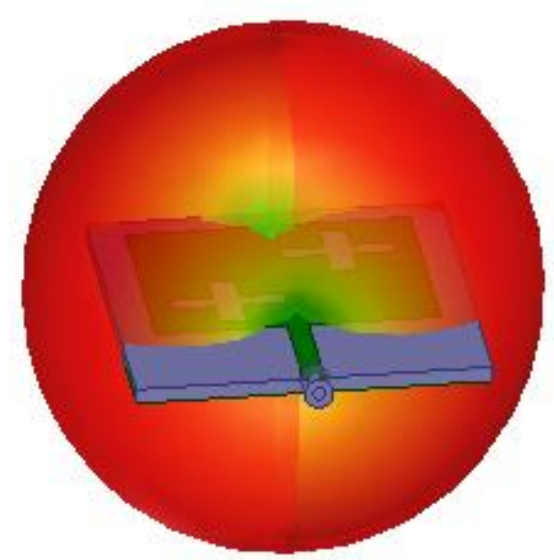

(c)

Figure 5. Simulated and the Measured Radiation Pattern of the Antenna (a) H-plane (b) E-plane (c) 3D Polar Plot of Radiation Pattern at $950 \mathrm{MHz}$ 
bandwidth additional parasitic capacitance is introduced by the fractional ground beneath the coplanar feeding line. Partial Ground results in enhancement of the lower frequency band. To reduce the Axial Ratio, two cross-shaped slots are incorporated diagonally into the rectangular patch. The orthogonal, equal magnitude current distribution is achieved by two means: I) by symmetrical Cross Shaped slots and II) by diagonally placing the identical slots. This results in the reduction in axial ratio and hence circular polarization is achieved. These plus-shaped slots introduce two orthogonal field components, and the symmetrical structure of the square patch and plus-shaped slots give in phase quadrature with equal amplitude. A prototype of the proposed antenna is fabricated and tested on the network analyzer, as shown in Figure 3. The S11 parameter of the antenna is measured on Vector Network Analyzer (VNA), as shown in Figure 4. It is evident that the antenna has $-10 \mathrm{~dB}$ impedance bandwidth from $700 \mathrm{MHz}$ to $2.9 \mathrm{GHz}$ and $-3 \mathrm{~dB}$ axial ratio bandwidth from $1.5 \mathrm{GHz}$ to $2.5 \mathrm{GHz}$. There is good agreement between simulated and measured S11 parameter and covering the entire GSM, UMTS, and LTE bands. The radiation pattern of the antenna is measured using Spectrum Analyzer, automated turnTable and data logger at $950 \mathrm{MHz}$, as shown in Figure 4. It is apparent that the antenna has an omnidirectional radiation pattern.

\section{Spectrum Occupancy Measurement}

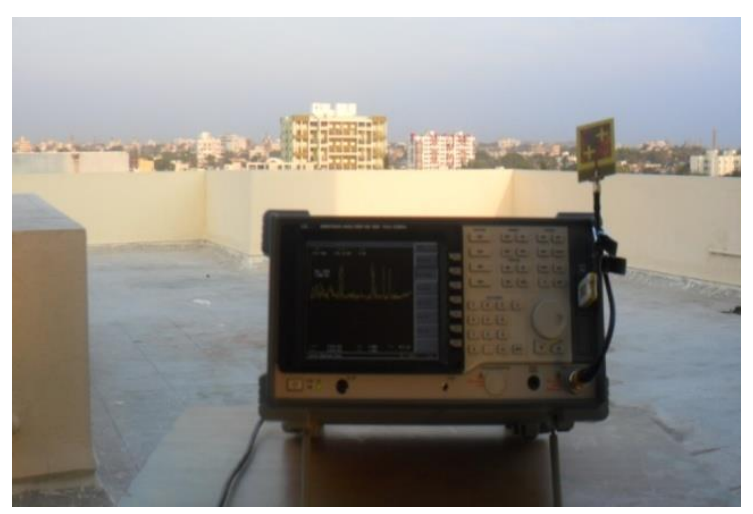

(a)

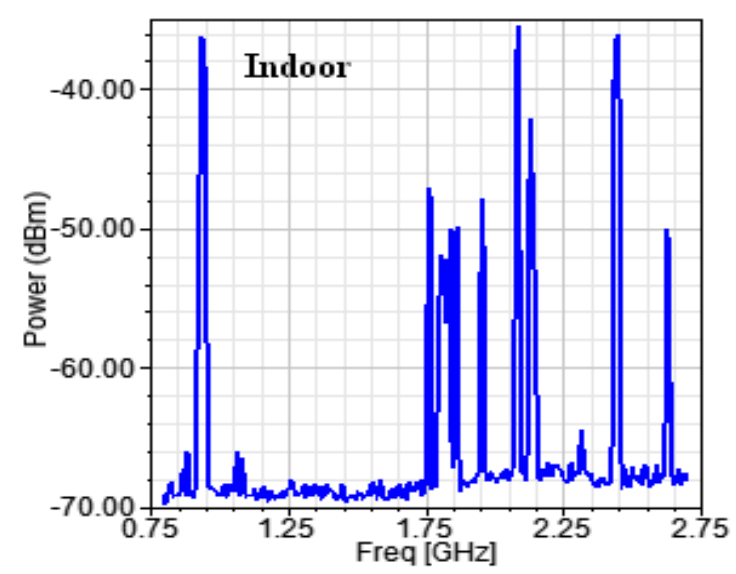

(b) 


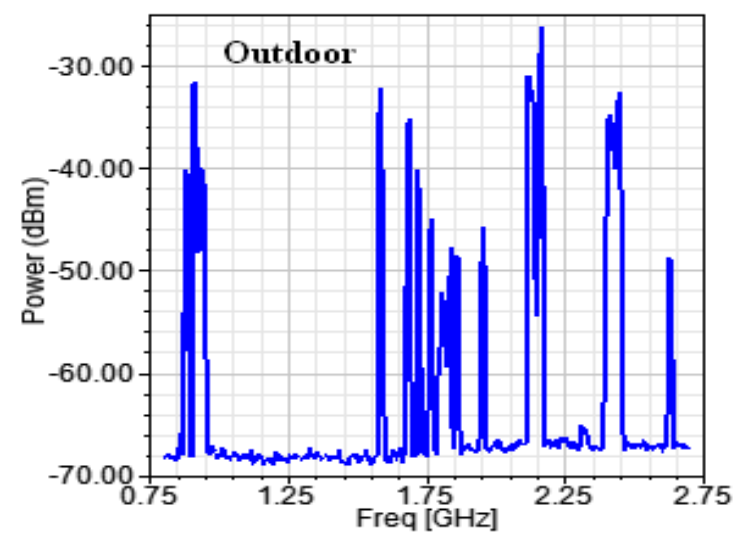

(c)

Figure 6. Actual Ambient Power Received by the Designed Antenna (a) Spectrum Occupancy Measurement Setup at Solapur, India (a) Indoor Surrounding (b) Outdoor Surrounding

The survey of the ambient power is done by the designed antenna in indoor as well outdoor surroundings. The outdoor spectrum occupancy measurement setup is shown in Figure 6(a). The measurement setup includes LIGNEX1 NS30A spectrum analyzer, Ntype to female SMA connector cable and the proposed antenna. The developed spectrum occupancy measurement setup is very simple and can be easily move from one location to another. The measurement is performed at Solapur, India. However, the setup is only intended to identify the spectrum sensing ability of the proposed antenna and a wellorganized measurement is required to conclude the spectrum occupancy in a specific region. The proposed antenna shows improved spectrum sensing ability over the antenna used in the existing literature, as shown in Figure 6. The measurement setup is inspired by [3-6]. The indoor surveying is done within a communication laboratory, and peak -31.5 $\mathrm{dBm}$ power is sensed at UMTS band. The outdoor surveying is done on the 3-story building roof-top and peak $-24 \mathrm{dBm}$ power is sensed at GSM and UMTS bands. Significant variations around $(8-10 \mathrm{dBm})$ are observed due to the multipath fading effect which may be very useful for channel estimation.

\section{Conclusion}

A circularly polarized wide axial ratio bandwidth microstrip monopole antenna is designed. The proposed antenna is fabricated, and measured results show good agreement with simulation results. The spectrum sensing ability of the proposed antenna is verified through the spectrum occupancy measurement setup, and results are found better than the antenna used in literature. The use of microstrip antenna in the spectrum occupancy measurement setup will reduce the system complexity, cost and the portability of the setup will be very easy.

\section{References}

[1] J. Mitola and G. Q. Maguire, "Cognitive Radio: Making Software Radios more Personal”, IEEE Personal Commun. Mag., vol. 6, no. 4, (1999), pp. 13-18.

[2] J. Mitola, "Cognitive Radio Architecture Evolution", Proceedings of the IEEE, vol. 97, no. 4, (2009), pp. 626-641.

[3] H. Marko, "Spectrum Occupancy Measurements: A Survey and Use of Interference", IEEE Commun. Surveys \& Tutorials, vol. 18, no. 4, (2016), pp. 2386-2414.

[4] W. Matthias, W. Jin and M. Petri, "Evaluation of Spectrum Occupancy in Indoor and Outdoor Scenario in the Context of Cognitive Radio", 2nd Int. Conf. Cognitive Radio Oriented Wireless Networks Commun., (2007). 
[5] F. Odufuwa, "Open Spectrum Development for Nigeria Case Study", Association for Progressive Communication, (2010).

[6] P. Kishor, E. S. Knud and P. Ramjee, "Stochastic Duty Cycle Model Based on Measurement for Cognitive Radio, 15th Int. Symposium Wireless Personal Multimedia Commun., (2012).

[7] N. Nasimuddin, N. Chen and Q. Xianming, "A Compact Circularly Polarized Cross-shaped Slotted Microstrip Antenna”, IEEE Trans. Antennas Propag., vol. 60, no. 2, (2012), pp. 1584-1588.

[8] C. A. Balanis, "Antenna Theory, Analysis and Design", Jhon Wiley \& Sons, Inc, New York, (1997).

[9] R. Garg, P. Bhartia, I. Bahl and A. Ittipiboon, "Microstrip Antenna Design Handbook", Artec House, Boston, (2001).

[10] C. Horng-Dean, C. Yen, S. Desmond and K. Shang-Huang, "Compact Broadband Dual Coupling-Feed Circularly Polarized RFID Microstrip Tag Antenna Mountable on Metallic Surface”, IEEE Trans. Antennas Propag, vol. 60, no. 12, (2012), pp. 5571-5577.

[11] U. Yu, N. Eisuke and A. Masayoshi, "Dual-Polarized Microstrip Array Antenna with Orthogonal Feed Circuit”, Proc. IEEE, AP-S/URSI, (2011).

[12] P. C. Sharma and S. G. Kuldip, "Analysis and Optimized Design of Single Feed Circularly Polarized Microstrip Antennas", IEEE Trans Antennas Propag., vol. AP-31, no. 6, (1983), pp. 949-955.

[13] I. Hisao, "A Circularly Polarized Small Size Microstrip Antenna with a Cross Slot", IEEE Trans. Antennas Propag., vol. 44, no. 10, (1996), pp. 1399-1401.

[14] W. Kin-Lu and F. L. Yi, "Circularly Polarized Microstrip Antenna With a Tuning Stub", IEEE Electronics Lett., vol. 34, no. 9, (1998).

[15] W. Kin-Lu and H. C. Ming, "Small Slot Coupled Circularly Polarized Microstrip Antenna with Modified cross-slot and bent tuning stub", IEEE Electronics Lett., vol. 34, no. 16, (1998).

[16] K. Jayendra, "Compact MIMO Antenna", Microwave Optical Technology Lett., vol. 58, no. 6, (2016), pp. 1294-1297.

[17] K. Jayendra, B. Banani, F. A. Talukdar and N. Arnab, "X-band Antenna Printed on Multilayered Substrate", IET Micro. Antennas Propag., (2017).

\section{Authors}

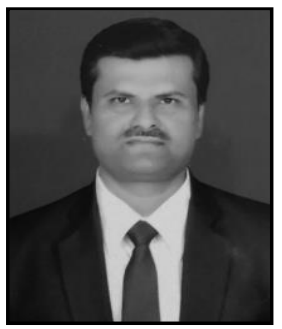

Siddharudha Shirgan has received ME degree and persuing $\mathrm{Ph.D.} \mathrm{in} \mathrm{Electronics} \mathrm{Engineering} \mathrm{from} \mathrm{Shivaji} \mathrm{University,}$ Maharashtra, India. He has total 17 years of experience in teaching. His research interests are on Cognitive Radio and Microstrip antennas. He has delivered guest lectures on Recent trends in communication technology and Microstrip antenna. He is a life member of Indian Society for Technical Education (ISTE).

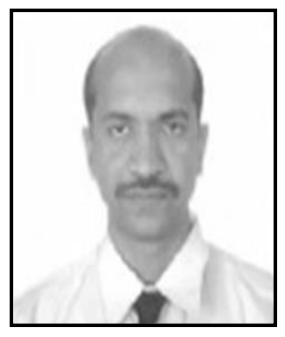

U. L. Bombale has received M.E in Electronics \& Telecommunication in 1994 from College of Engineering, Pune, And $\mathrm{PhD}$ from the Dhirubhai Ambani Institute of Information \& Communication Technology (DA-IICT) Gandhinagar, Gujarat, India, under the guidance of Dr. Sanjeev Gupta. Currently he is working as a Prof. in Dept. of Technology, Shivaji university, Kolhapur (India).He has total 24 years of experience in teaching. His research interests are on Microstrip antennas, Smart antennas and Cognitive Radio. He has deleivered expert lectures on Microstrip antenna, Microwave Devices and Amplifier Design, High speed Analog and Digital Design etc. 